\title{
Upper-bound general circulation of coupled ocean-atmosphere: Part 1. Atmosphere
}

\author{
Hsien-Wang Ou* \\ Department of Earth and Environmental Sciences, Lamont-Doherty Earth Observatory of Columbia \\ University, Palisades, NY 10964, United States
}

\section{A R T I C L E I N F O}

\section{Article history:}

Received 11 December 2012

Received in revised form 2 September 2013

Accepted 9 September 2013

Available online 25 September 2013

\section{Keywords:}

General atmosphere circulation

Maximum entropy production

Potential vorticity mixing

Meridional cells

Extratropical tropopause

\begin{abstract}
A B S T R A C T
We consider the general atmospheric circulation within the deductive framework of our climate theory. The preceding three parts of this theory have reduced the troposphere to the tropical and polar air masses and determined their temperature and the surface latitude of their dividing boundary, which provide the prior thermal constraint for the present dynamical derivation. Drawing upon its similar material conservation as the thermal property, the (columnar) potential vorticity (PV) is assumed homogenized as well in air masses, which moreover has a zero tropical value owing to the hemispheric symmetry. Inverting this PV field produces an upperbound zonal wind that resembles the prevailing wind, suggesting that the latter may be explained as the maximum macroscopic motion extractable by random eddies - within the confine of the thermal differentiation.

With the polar front determined in conjunction with the zonal wind, the approximate leveling of the isobars at the surface and high aloft specifies the tropopause, which is colder and higher in the tropics than in the polar region. The zonal wind drives the meridional circulation via the Ekman dynamics, and the preeminence of the Hadley cell stems from the singular Ekman convergence at the equator that allows it to supply the upward mass flux in the ITCZ demanded by the global energy balance.
\end{abstract}

(c) 2013 Elsevier B.V. All rights reserved.

\footnotetext{
Lamont-Doherty Earth Observatory Contribution Number 7726.

* Correspondence to: Lamont-Doherty Earth Observatory of Columbia University, 61 Route 9W, Palisades, NY 10964, United States. Tel.: +1 9174779985.

E-mail address: dou@ldeo.columbia.edu
} 


\section{Introduction}

While the atmospheric motion is highly complex, spanning all spatiotemporal scales, it exhibits nonetheless persistent large-scale structure, which with varying scope falls under the rubric of the "general atmospheric circulation (GAC)". The attempts to understand the phenomenon have propelled much progress in dynamical meteorology, but despite great strides made to date, our explanations of the GAC remain deeply incongruous. For the tropical circulation, for example, one often invokes the thermal-direct Hadley cell, which drives the upper-level wind via the angular momentum conservation (Hadley, 1735; Held and Hou, 1980). Such conservation is predicated on the absence of eddies (the axisymmetric regime) and yet it is precisely the eddy-induced momentum flux that is commonly ascribed in the generation of the polar jet stream (Starr, 1968; Held and Hoskins, 1985).

There is no evidence that eddies are less important in the tropics, and in fact the eddy diffusivity diagnosed from general circulation models (GCMs) is largest in the upper tropical troposphere (Plumb and Mahlman, 1987) just where the axisymmetry is assumed. It is the global import of eddies that called into question Hadley's original exposition of the easterly trades and propelled the view that the eddy transport of angular momentum holds the key to our understanding of the GAC (Jeffreys, 1926; Rossby, 1947; Lorenz, 1967). This prescription remains unfulfilled - a shortfall that has perhaps contributed to the recent revival of the axisymmetric regime, which is not based on scaling argument but its amenability to mathematical treatment (Held and Hou, 1980).

The eddy-driving of the polar jet stream is a well-studied problem, but the mechanistic approach remains qualitative and often diagnostic (Held and Hoskins, 1985). This is due in part to the vagary of eddies but more fundamentally to the non-conservation of the angular momentum so even the sign of its flux cannot be ascertained (Starr, 1968). This difficulty can be partly overcome by considering the eddy flux of potential vorticity (PV), which can only be down-gradient because of its material conservation; and moreover if one were to parameterize this flux by the eddy diffusivity, the latter, being a manifestation of the microscopic motion (Taylor, 1915), should be the same as that operates on the thermal field. Exploiting this thermal/dynamical symmetry, Green (1970) has reproduced certain features of the GAC, but he left unanswered what determines the thermal field and its implied eddy diffusivity.

It is seen therefore that the GAC may be explained only in conjunction with the thermal field hence only within the thermodynamical closure of the global system. The formulation of such a closure has been the ongoing effort of this author with the stated aim of deriving the earth's generic climate forced only by the solar insolation. The logical steps taken so far (Ou, 2001, 2006, 2007, referred as Ou01, Ou06 and Ou07, respectively) have led to the determination of certain thermal properties, which, having invoked only thermodynamics, may justifiably be posed as given for the present dynamical derivation. In this two-part paper, we shall consider the general circulation of first the atmosphere and then the ocean, which would complete our climate theory.

For the organization of this part 1 on the GAC, we first rehash in Section 2 the key closure assumption of the theory, namely the maximized entropy production (MEP), and the thermal properties determined previously that serve as the prior constraint. We then deduce in Section 3 the PV configuration and discuss its supporting evidence. We invert this PV in Section 4 to obtain the upper-bound zonal wind and compare it with observations. In Sections 5 and 6, we derive auxiliary GAC properties pertaining to the tropopause height and the meridional circulation, respectively. In Section 7, we summarize the main findings and discuss their implications - the latter particularly in their contrast with the existing views of the GAC.

\section{MEP and prior thermal constraint}

Our consideration of the GAC represents a logical progression of our climate theory whose closure invokes the MEP. Although this principle does not enter our dynamical derivation, we shall nonetheless reiterate our perspective of the MEP in view of the recent discussions in the literature.

Following Paltridge's (1975) initial application of the MEP to the climate problem, its physical foundation has gradually strengthened through the years (Ozawa et al., 2003; Martyushev and Seleznev, 2006; Kleidon, 2009), including, particularly notably, its linkage to the information theory (Dewar, 
2003). Although Dewar's derivation was later faulted (Grinstein and Linsker, 2007), his interpretation further enhances our perspective of the MEP as rooted in the turbulence of the climate system (Ou06): the microscopic nondeterminism of turbulence amounts to the missing information and the macroscopic reproducibility of the climate would be propelled by the maximization of the information entropy (Dewar, 2005). Being predicated on the turbulence, our narrow application of the MEP to the climate system thus cannot be refuted by consideration of non-turbulent or linear-response systems (Prigogine, 1955; Bruers, 2006; Li, 2009). In addition, it is often overlooked that the MEP implies the second law under equilibrium (Martyushev and Seleznev, 2006) hence represents a palpable generalization of the second law to the nonequilibrium thermodynamics - an attribute missing from other extremization schemes (for example, Bruers, 2006).

With the above perspective of the MEP, we shall next clarify some salient points in its application. First, since the MEP is a selection rule, it can be applied hierarchically, which justifies our deduction of first the global-mean temperature (Ou01) and then the meridional thermal field (Ou06); this approach is additionally supported by recent findings that the entropy productions are separately maximized by vertical and horizontal processes (Lucarini et al., 2011; Pascale et al., 2012). Second, since the turbulence is internal to the fluid, the MEP should apply to the atmosphere and ocean concurrently, which has obviated the need of extraneous closure assumptions (Paltridge, 1975; Noda and Tokioka, 1983; Obrien and Stephens, 1995) and simply resolved the system-boundary ambiguity (Virgo, 2010). Third, being a selection rule, the MEP is subjected to all veritable constraints, including the dynamical ones (Goody, 2007), so there is no presumption that the prior thermal constraint would not be altered by the dynamical consideration (see further discussion in Section 7.1). Fourth, since the MEP is applied through the boundary entropy flux, it has already accounted for all internal irreversible processes, including the frictional dissipation on the GAC (Peixoto et al., 1991; Pauluis and Held, 2002); the latter thus has no import on the prior thermal constraint.

For self-containment, we shall next recount the logical steps leading to the prior thermal constraint. We determined in Ou01 the global-mean temperature, which then constrains the meridional thermal field derived in Ou06. In the latter, we first distinguished the forced- and free-convective regimes to divide the ocean into warm and cold water masses separated by an outcropped boundary; we then applied the atmospheric MEP to reduce the troposphere to two thermal masses as well with their boundary aligned with the ocean front. The thermal configuration is as sketched in Fig. 1 whose internal boundaries are well discerned in observations: the oceanic boundary being the permanent thermocline outcropped as the subtropical front that separates the warm and cold water spheres, and the atmospheric boundary being the polar front that divides the tropical and polar air masses. As the last step in Ou06, we applied the oceanic MEP to determine the temperature of all four thermal masses and the surface latitude of their dividing boundaries; these constitute the prior thermal constraint of the present dynamical derivation.

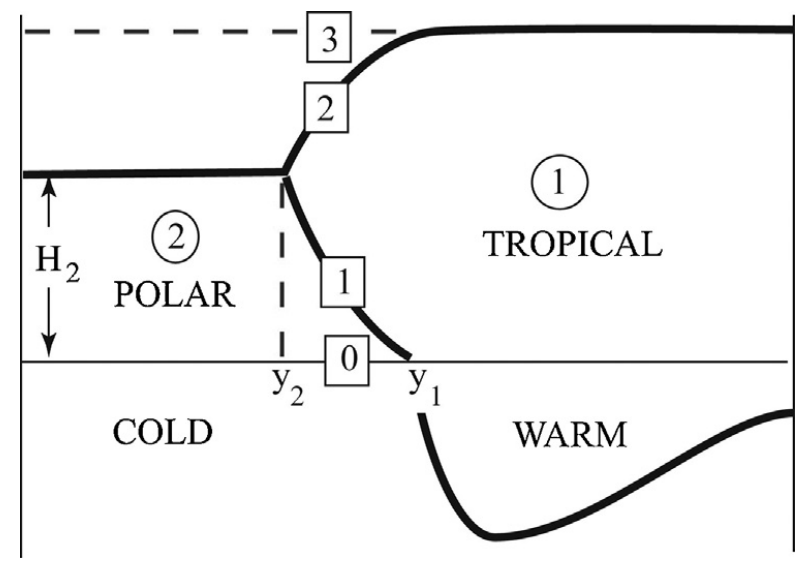

Fig. 1. The thermal configuration of the model climate. The circled numbers indicate air masses ( 1 for tropical and 2 for polar) and the numbers in squares are for different levels ( 0 for the surface, 1 for the polar front, 2 for the tropopause, and 3 for the highest level reached by the tropopause). The prior thermal constraints are the temperature of the fluid masses and the surface latitude of their dividing boundaries $\left(y_{1}\right)$. 


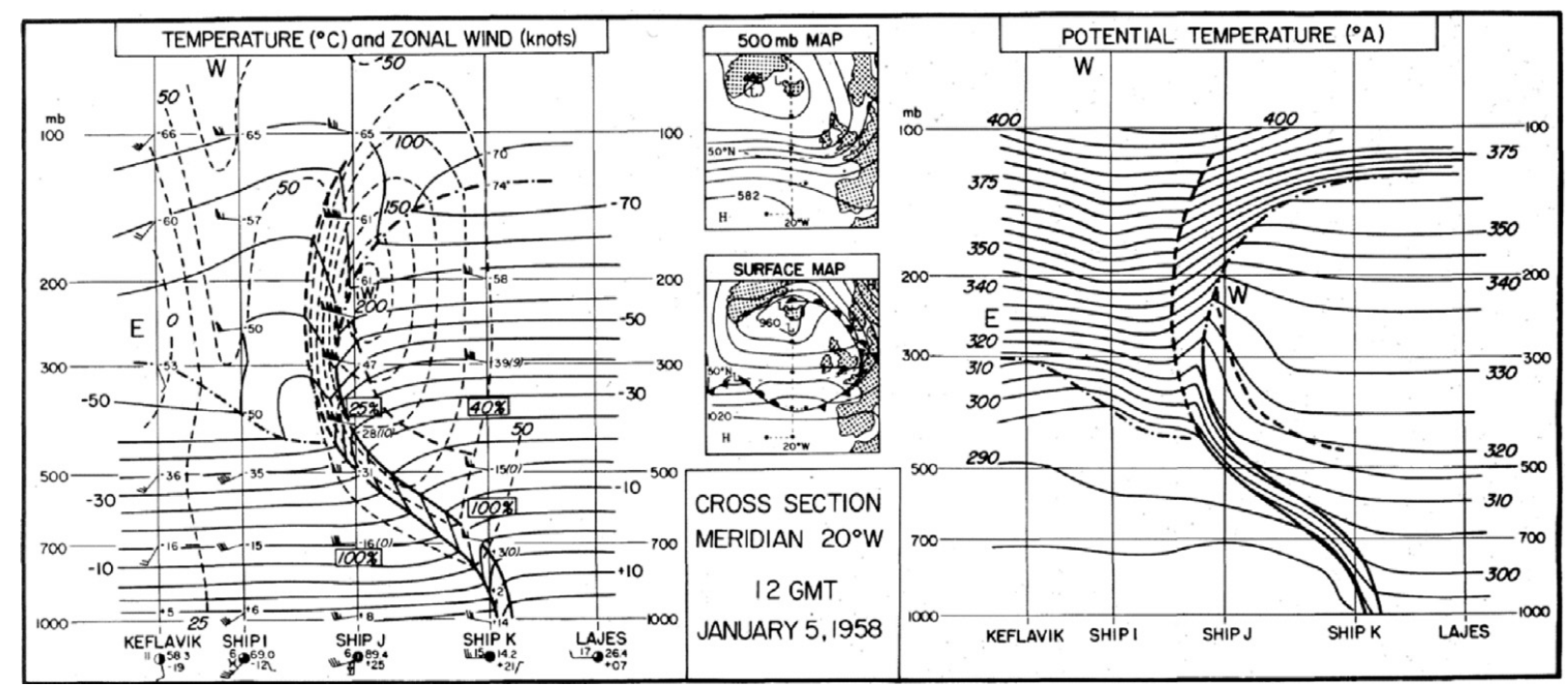

Fig. 2. A meridional section across the polar front (heavy solid). Left: isotherms (solid, in degrees C) and isotachs (dashed, in knots) with the tropopause indicated in dash-dotted lines; right: potential temperature (in degrees K) (reproduced from Fig. 7.5 of PN69).

With the polar front constituting a thermal boundary hence impacting the GAC, its observational basis merits further examination. For this, we first note that the polar front is arguably the most pronounced large-scale structure in synoptic maps, whose prominence has spawned the Norwegian school of polar-front theories (see Palmen and Newton, 1969, hereafter PN69); but this early flourish aside, the polar front is rarely mentioned in current climate discussions. We attribute this seeming disconnect to the fact that the climatic state is usually defined by the Eulerian zonal mean, which exhibits barely discernible polar front (Peixoto and Oort, 1992, henceforth P092) because of its extreme latitudinal excursion (PN69). Since the zonal averaging is merely a descriptive device, it obviously is unjustified if it erases this distinct internal boundary. Rather, the proper averaging should be the Lagrangian type that preserves this boundary - while acknowledging its undulation due to processes, such as hydrodynamic instability and orographic steering, that are peripheral to the genesis of the GAC. With this perspective, we reproduce in Fig. 2 a synoptic meridional section (PN69, their Fig. 7.5), which thus may serve as a baseline observation for our model climate.

\section{PV homogenization}

Since the eddy diffusivity is a manifestation of the microscopic motion (Taylor, 1915), its value inferred from the macroscopic thermal field should operate on the conservative dynamical properties as well. While the isentropic PV (IPV) has been widely used as a conservative tracer in synoptic studies (Hoskins et al., 1985), it is inadequate for constraining the GAC since it is premised on isentropic motion, which does not hold in the frontal zone where the isentropes intersect the quasi-material boundaries. As an alternative to the IPV, we shall first show that the eddy motion within air masses is barotropic to allow the definition of a columnar PV (or PV for short), and this columnar PV is globally conserved as it properly accounts for the vortex stretching in the frontal zone.

The thermal property that is materially conserved hence homogenized in air masses is the moiststatic energy (E) so that (all symbols are conventional, see PO92, Chapter 3)

$$
\delta E \approx c_{p} \delta T-\frac{R_{d} T}{p} \delta p+\frac{.622 L}{p} \delta e \approx 0,
$$

where $\delta$ indicates the spatial variation of the macroscopic fields. As alluded to in Ou07, since the subsidence that spans the bulk of the global domain is relatively weak, the above homogenization involves three processes: the deep updraft that propels the moist static energy to a constant saturation value; the lateral mixing in the ambient air by eddies; and the constant offset of the ambient vapor pressure from its saturation value. The last is a pronounced feature in observations (Webster, 1994), which has 
been subjected to a plausible explanation by Ou07 hence may be regarded as a deductive property. It implies that the variation of the ambient vapor pressure is governed by the Clausius-Clapeyron equation hence

$$
\delta e \approx \delta e_{s}=\frac{T_{c} e_{s}}{T^{2}} \delta T,
$$

where the suffix " $s$ " stands for "saturation" and $T_{c} \equiv 0.622 L / R_{d}$. Substituting (3.2) into (3.1), the latter can be cast in the form

$$
\Gamma(T, p) \delta T \approx \delta p,
$$

where $\Gamma$ is a function only of $T$ and $p$. With this equation, it is seen that the temperature is unvarying on isobars, which implies the same for the density on account of the ideal-gas law.

Employing the Boussinesq approximation, the vorticity equation is (PO92, their Eq. (3.37)):

$$
\frac{d \vec{\omega}}{d t}-\vec{\omega} \cdot \nabla \vec{u}=-\nabla \frac{1}{\rho_{a}} \times \nabla p,
$$

where $\vec{\omega} \equiv \hat{k} f+\nabla \times \vec{u}$ is the total vorticity, $f \equiv 2 \Omega \sin \phi$ is the Coriolis parameter with $\Omega$, the earth's rotation rate and $\phi$, the latitude, and $\rho_{a}$ is the air density. With the foregoing alignment of the pressure and density surfaces, the solenoidal term (the rhs) vanishes, which implies that the quasi-geostrophic motion characterizing eddies has no vertical shear. Near the equator where the geostrophy breaks down, the vertical shear can be removed by the deep convection in the Intertropical Convergence Zone (ITCZ), so together one may assume the eddy motion to be columnar in air masses (Schneider, 2006, his Section 3.2). This deduction is consistent with the observed vertical orientation of the constant angular-momentum lines in the tropics (Schneider, 2006, his Fig. 1c), and it does not contradict the strong baroclinicity of the zonal wind in the extratropics since it allows vertical shear across the polar front.

For the columnar motion, the relative vorticity has only vertical component, which moreover is vertically unvarying, one thus may integrate the vertical component of (3.4) through the depth of the air mass bounded by quasi-material surfaces, applying the frictional torque if the air column abuts the surface and neglecting the interfacial stress, to yield

$$
\frac{d P}{d t}=-\frac{\alpha}{h} \hat{k} \cdot \nabla \times \frac{\vec{u}}{h},
$$

where

$$
P \equiv \frac{f+\zeta}{h}
$$

is the (columnar) PV with $h$ being the layer thickness, $\varsigma$, the vertical component of the relative vorticity, $\alpha \equiv C_{d}\left|u^{\prime}\right|$ is a resistance coefficient with $C_{d}$ being the drag coefficient and $\left|u^{\prime}\right|$ the turbulent wind. We see that the vorticity equation is reduced to that of the shallow water even with the presence of moisture - despite conjecture to the contrary (Schneider, 2006).

Eq. (3.5) states that the PV is materially conserved, so the eddy mixing would smooth its macroscopic representation, just as the thermal field (3.1). To formalize this statement, we first recast (3.5) in its flux form with the aid of the continuity equation:

$$
(h P)_{t}+\nabla \cdot(h \vec{u} P)=-\alpha \hat{k} \cdot \nabla \times\left(\frac{\vec{u}}{h}\right),
$$

and then apply the Reynolds decomposition:

$$
P \equiv \bar{P}+P^{\prime}, \text { etc. },
$$

where overbars denote the eddy-averaged (macroscopic) fields assumed time- and zonal-invariant, and the primes are microscopic perturbations associated with eddies. Averaging (3.7) over eddies, 
neglecting the depth perturbations, and integrating the resulting equation from the pole where the eddy-induced PV transport and mean zonal wind vanish, we obtain, for the polar air mass,

$$
\bar{h} \overline{v^{\prime} P^{\prime}}=\frac{\alpha \bar{u}}{\bar{h}},
$$

which states that the PV transport at a given latitude (the $l h s$ ) is balanced on the rhs by the net frictional torque acting on the air poleward of that latitude. For the tropical air mass, the integration commences at its poleward tip (see Fig. 1) where the PV transport and surface stress are again zero, and we obtain an equation similar to (3.9) except the rhs would be zero in the frontal zone (since it is not abutting the surface). Given the material conservation of the PV (3.5), its eddy-induced transport can be linked to the mean gradient via

$$
\overline{v^{\prime} P^{\prime}} \equiv-\frac{k}{R} \cdot \frac{d \bar{P}}{d \phi}
$$

where the eddy diffusivity $k$ is the same as that mixing the thermal field (Section 1 ). And with the thermal configuration shown in Fig. 1, the eddy diffusivity is asymptotically large in air masses but small at the internal (quasi-material) boundaries. Substituting (3.10) into (3.9), non-dimensionalizing the variables by scales denoted by brackets below, and dropping the overbars hereafter, we obtain

$$
\left(1-y^{2}\right) P_{y}=\frac{\hat{\alpha} m}{h^{2}}
$$

where $y \equiv \sin \phi$ and its subscripted use denotes a derivative

$$
P \equiv h^{-1}\left(y-m_{y}\right)
$$

is the columnar PV from (3.6) scaled by $[P]=2 \Omega / H_{2}$ (see footnote ${ }^{1}$ ), $m \equiv u \cos \phi$ is the relative angular momentum scaled by $[\mathrm{m}]=2 \Omega R^{2}$, the zonal wind by $[u]=2 \Omega R$, the layer depth by $[h]=H_{2}$, and the dimensionless parameter $\hat{\alpha} \equiv\left(k \mathrm{H}_{2}\right)^{-1} \alpha R^{2}$ is a measure of the importance of the surface friction relative to the lateral eddy mixing.

With the eddy diffusivity being asymptotically large in air masses, (3.11) implies $P_{y} \approx 0$ or PV is homogenized in air masses. While its value in the polar air mass is yet to be determined, its value in the tropical air mass would be zero on account of the hemispheric symmetry. ${ }^{2}$ Is this two-banded PV supported by observation? As alluded to earlier, one needs to apply the Lagrangian averaging to preserve the internal boundary and observational analysis of this sort has been carried out by Platzman (1949) and Swanson (2001) for the IPV in the middle-world (Hoskins, 1991). They both show two homogeneous IPV bands bisected by the extratropical tropopause (ET) with near-zero tropical value, in consistency with our deduced configuration for the columnar PV. ${ }^{3}$ Also in support of the quasi-material ET, the eddy diffusivity diagnosed from observations shows a distinct minimum there (Haynes and Shuckburgh, 2000).

We have neglected the rhs of (3.11) in the asymptotic limit, an approximation that can be assessed from observations. Using $C_{d} \approx 10^{-3},\left|u^{\prime}\right| \approx 10 \mathrm{~m} / \mathrm{s}$, we have $\alpha \approx 10^{-2} \mathrm{~m} / \mathrm{s}$; setting additionally $k \approx 5 \times 10^{6} \mathrm{~m}^{2} \mathrm{~s}^{-1}$ (Haynes and Shuckburgh, 2000; Plumb and Mahlman, 1987), a polar tropopause height of $10 \mathrm{~km}$ and typical zonal wind strength of $20 \mathrm{~m} / \mathrm{s}$, and recalling that the zonal wind has been scaled by the earth's surface speed at the equator $(873 \mathrm{~m} / \mathrm{s})$, the rhs is much smaller than unity, which thus may explain why the observed PV is strongly homogenized in air masses.

\section{Zonal wind}

Since without eddy mixing, (3.11) yields a vanishing GAC, the PV homogenization represents the other extreme of the maximum - hence "upper-bound" - wind. The primary inquiry here is whether

\footnotetext{
1 The polar tropopause height $H_{2}$ will be determined in Section 5, which is largely independent of the GAC.

2 Since the seasonal migration of the ITCZ amounts to cross-equatorial mixing (Plumb and Mahlman, 1987), there can be no PV front at the equator.

${ }^{3}$ Because of lack of baroclinicity outside the frontal zone, the uniform IPV in the polar stratosphere implies uniform columnar $\mathrm{PV}$ in the underlying polar air mass.
} 
this upper-bound wind may capture the essential character of the observed wind so as to offer a plausible explanation.

The calculation of the zonal wind involves the inversion of the PV of a baroclinic fluid. With the tropical and polar air masses designated respectively by subscripts 1 and 2 , and $\left[y_{1}, y_{2}\right]$ marking the frontal zone (only $y_{1}$ is known from the prior thermal constraint), the deduced PV configuration implies that, from (3.12)

$$
y-\frac{d m_{1}}{d y}=0 \text { for } y \leq y_{2},
$$

and, with the unknown $P_{2}$,

$$
y-\frac{d m_{2}}{d y}=P_{2} h_{2} \text { for } y \geq y_{1} .
$$

In the frontal zone, $h_{2}$ is coupled to the zonal wind via the Margules equation or dimensionally

$$
f\left(u_{1}-u_{2}\right)=\frac{g^{\prime}}{R} \cdot \frac{d h_{2}}{d \phi},
$$

where

$$
g^{\prime} \equiv g \frac{\Delta T}{T_{2}}
$$

is the reduced gravity under the Boussinesq approximation with $\Delta T \equiv T_{1}-T_{2}$. To see that the reduced gravity can be assumed uniform along the front, we approximate the lapse rate by a constant $\gamma$ $(\approx 6.5 \mathrm{~K} / \mathrm{km})$, so the hydrostatic balance and ideal-gas law combine to yield, within air masses,

$$
d \ln p=g\left(\gamma R_{d}\right)^{-1} d \ln T
$$

Integrating this equation from the surface up in both air masses and matching the pressure at the surface and across the frontal interface then yields

$$
\frac{\Delta T}{T_{2}}=\frac{\Delta T_{0}}{T_{2,0}},
$$

where the subscript " 0 " denotes the surface value. With this, the reduced gravity (4.4) thus is uniform and given by its surface value known from the prior thermal constraint. Applying the scaling rules to (4.3), it is nondimensionalized to

$$
y\left(m_{1}-m_{2}\right)=\varepsilon\left(1-y^{2}\right) \frac{d h_{2}}{d y} \text { for } y_{2}>y>y_{1},
$$

where $\varepsilon$ is the Burger number defined by

$$
\varepsilon \equiv\left(\frac{R_{c}}{R}\right)^{2}
$$

with $R_{c} \equiv\left(g^{\prime} H_{2}\right)^{1 / 2}(2 \Omega)^{-1}$ being the baroclinic deformation radius. We have thus three first-order ordinary differential equations governing three variables $\left(m_{1}, m_{2}, h_{2}\right)$, and given the unknown values of $P_{2}$ and $y_{2}$, the problem contains five degrees of freedom, and the five constraints needed for the closure are the following: we have by definition

$$
h_{2}=0 \text { at } y=y_{1} ;
$$

and by scaling

$$
h_{2}=1 \text { for } y \geq y_{2} \text {; }
$$

on account of the spherical geometry, the zonal wind vanishes at the pole

$$
m_{2}=0 \text { at } y=1 \text {; }
$$


and because of the approximate stationarity, we have the well-known constraint that the net surface torque is zero (PO92, Section 11.2.2):

$$
\int_{0}^{1} m d y=0
$$

the last required condition can be discerned from (3.11) that at the foot of the front where $h_{2}$ vanishes, we have

$$
m_{2}=0 \text { at } y=y_{1} \text {; }
$$

since the eddy diffusivity, while asymptotically large, remains finite. Indeed the observed zonal wind at the surface drops sharply as one crosses over to the poleward side of the polar front (PN69, their Fig. 8.3), in support of this boundary condition.

With the problem closed, one may solve for the zonal wind using the method described in Appendix A. The solution depends on only two dimensionless parameters: the frontal position $y_{1}$ and the Burger number $\varepsilon$, both are known from the prior thermal constraint. If one uses a surface temperature difference of $20 \mathrm{~K}$ across the front and a polar tropopause height of $10 \mathrm{~km}$, the baroclinic deformation radius would be $600 \mathrm{~km}$ hence the Burger number is 0.01 . Setting additionally $y_{1}=0.5$, the solution for the zonal wind (solid lines) and interfacial height (dashed line) are plotted in Fig. 3 with the shade marking the frontal zone.

It is seen that the zonal wind exhibits a latitudinal pattern that resembles the prevailing wind as labeled: there are easterly trade, mid-latitude surface westerly, a weak polar easterly, and a strong polar jet stream above the polar front. Quantitatively, they have maximum speed of 40, 30, 160 and $15 \mathrm{~m} \mathrm{~s}^{-1}$, respectively, which are consistent with being upper bounds of the observed wind, but they are nonetheless of commensurate strength (see Fig. 8.3 of PN69) to support their practical relevance.

With the above, we see that the mere presence of a polar front bisecting homogeneous PV bands may capture the essential character of the GAC; the latter thus may be simply explained as follows: In the tropical air mass of zero PV, the zonal wind has zero shear hence a negative maximum at the equator (the easterly trade); the shear becomes increasingly negative poleward due to the beta effect, so the zonal wind attains a positive maximum (the jet stream) at its northern tip. In the polar air mass, the wind is zero at the foot of the front (4.13) with a strong negative sheer (equaling $-f$ ). As one proceeds poleward, the shear first increases to a positive value in the frontal zone due to the vortex stretching before it decreases owing to the beta effect; this shear distribution results in a zonal wind that has positive maximum in the frontal zone (the surface westerly), which may be reversed (the polar easterly) before it vanishes at the pole.

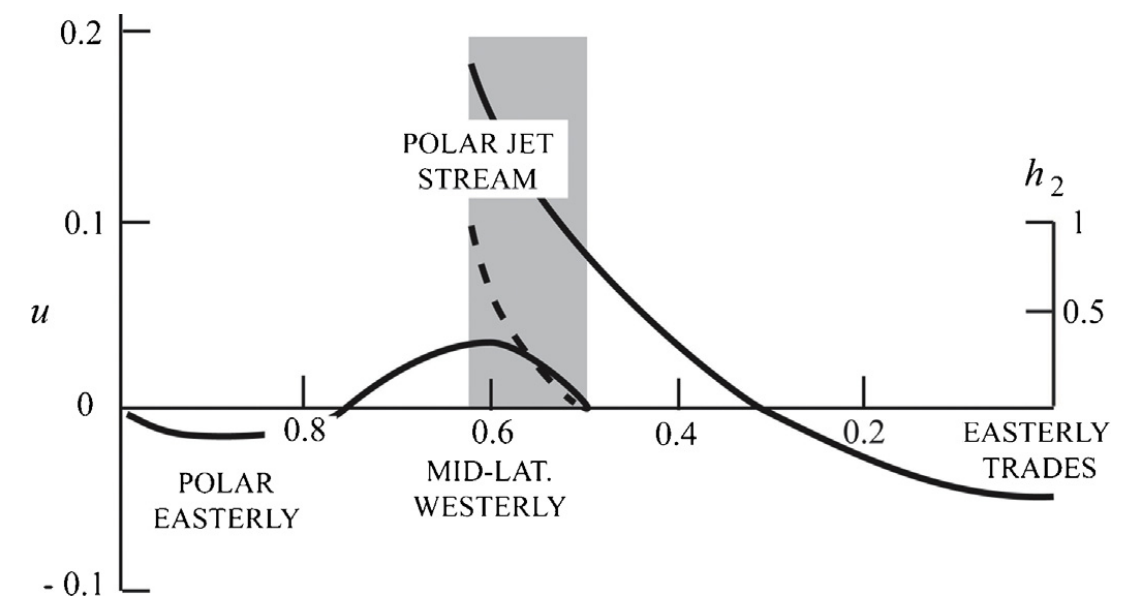

Fig. 3. The zonal wind of the two air masses (solid) and frontal height $h_{2}$ (dashed) for the case of $y_{1}=0.5$ and $\varepsilon=0.01$; the shade marks the frontal zone. The wind speed has been nondimensionalized by the earth's surface speed at the equator $(\approx 873 \mathrm{~m} / \mathrm{s})$ and the frontal height by the polar tropopause height. The corresponding prevailing winds are labeled. 


\section{Tropopause height}

In Fig. 1, we have assumed the tropopause to be higher in the tropics and we have used the polar tropopause height as the vertical scale in nondimensionalization, so for the dynamical closure, we need to determine the tropopause height, as considered here. In addition, what controls the height of the extratropical tropopause (ET) is a widely discussed subject in the literature and our theory offers a contrasting and simpler interpretation.

By way of argument, we begin with a horizontally uniform atmosphere devoid of the GAC. Since there is no moisture hence vanishing long-wave opacity above the tropopause, the latter is at the planetary skin temperature (Hartmann, 1994, Section 3.8) whose height thus is specified by the surface temperature and the lapse rate (Held, 1982) - both are deductively known (Ou01, Ou07). For a crude test, if one uses a planetary albedo of 0.3 (PO92, Fig. 6 .3), the planetary skin temperature would be $215 \mathrm{~K}$ (Hartmann, 1994); setting the surface temperature at $288 \mathrm{~K}$ and a lapse rate at $6.5 \mathrm{~K} \mathrm{~km}^{-1}$ then yields a tropopause height of $11 \mathrm{~km}$, which is within its observed range.

With the thermal differentiation of the tropical and polar air masses, so should their capping tropopause, which is subjected additionally to dynamical constraints. As a useful analogy, the configuration shown in Fig. 1 can be representative of the geostrophically adjusted state of three immiscible fluid masses (see for example, Cushman-Roisin, 1994, his Fig. 13-4d) corresponding in descending density to the polar troposphere, the tropical troposphere and the stratosphere. If the zonal flow is sufficiently weak at the surface so that the pressure is equalized, then subjected also to the vanishing pressure above the fluid, the medium-density mass would wedge between the other two masses, similar to that of Fig. 1.

The actual atmosphere is more complicated as the temperature and density vary vertically in air masses, but similar pressure equalizations hold: at the surface, even the upper-bound GAC only slightly perturbs the pressure in comparison with the atmospheric weight; and high aloft, the pressure eventually equilibrates as air becomes increasingly rarefied. Now that the pressure drops more rapidly with height in the denser polar than the tropical air masses and in the troposphere of finite lapse rate than the isothermal stratosphere, one sees immediately that the polar air mass must terminate at a lower altitude and higher temperature than the tropical air mass.

To facilitate a quantitative discussion, we provide the mathematical derivation in Appendix B, which allows first the calculation of tropopause properties outside the frontal zone and then the temperature and pressure profiles throughout the troposphere, including the frontal zone. The latter requires the input of the height distribution of the polar front (PF) from Section 4, which then specifies the ET via the hydrostatic balance. For simplicity, we have assumed the frontal zone to be narrow (relative to the equator-to-pole distance) and that, to be checked a posteriori, the pressure approximately equalizes at the highest reach of the tropopause.

An example of the solution is shown in Fig. 4 for which we have used the foregoing planetary skin temperature of $215 \mathrm{~K}$, surface temperature of the tropical and polar air masses at 290 and $270 \mathrm{~K}$, respectively, and the PF is as that shown in Fig. 3. The dashed line marks the tropopause and we have magnified the PF and ET to aid the visualization. It is seen that despite the extreme simplification of the model, the solution bears close resemblance to the observed fields (Fig. 2). Specifically, the temperature of the tropical and polar tropopause are 194 and $218 \mathrm{~K}$, their height, 14.8 and $8 \mathrm{~km}$, and their pressure, 120 and $320 \mathrm{mb}$, respectively, all are comparable with the observed values. Given the low pressure at the tropical tropopause, it justifies the pressure equalization at this level, as alluded to above. One notable feature is the inversion at the tropopause, which is particularly pronounced over the tropics. In the model, this is a consequence of the geostrophic adjustment, which requires the stratosphere at the PF/ET juncture to be no denser than the adjoining tropical air mass (Appendix B); the model thus may explain the cold trap in the tropics even without the ozone absorption in the stratosphere.

\section{Meridional cells}

The zonal wind determined in Section 4 pertains to that in the free troposphere, which would drive a meridional wind in the surface Ekman layer and hence meridional cells via continuity. For simplicity, 

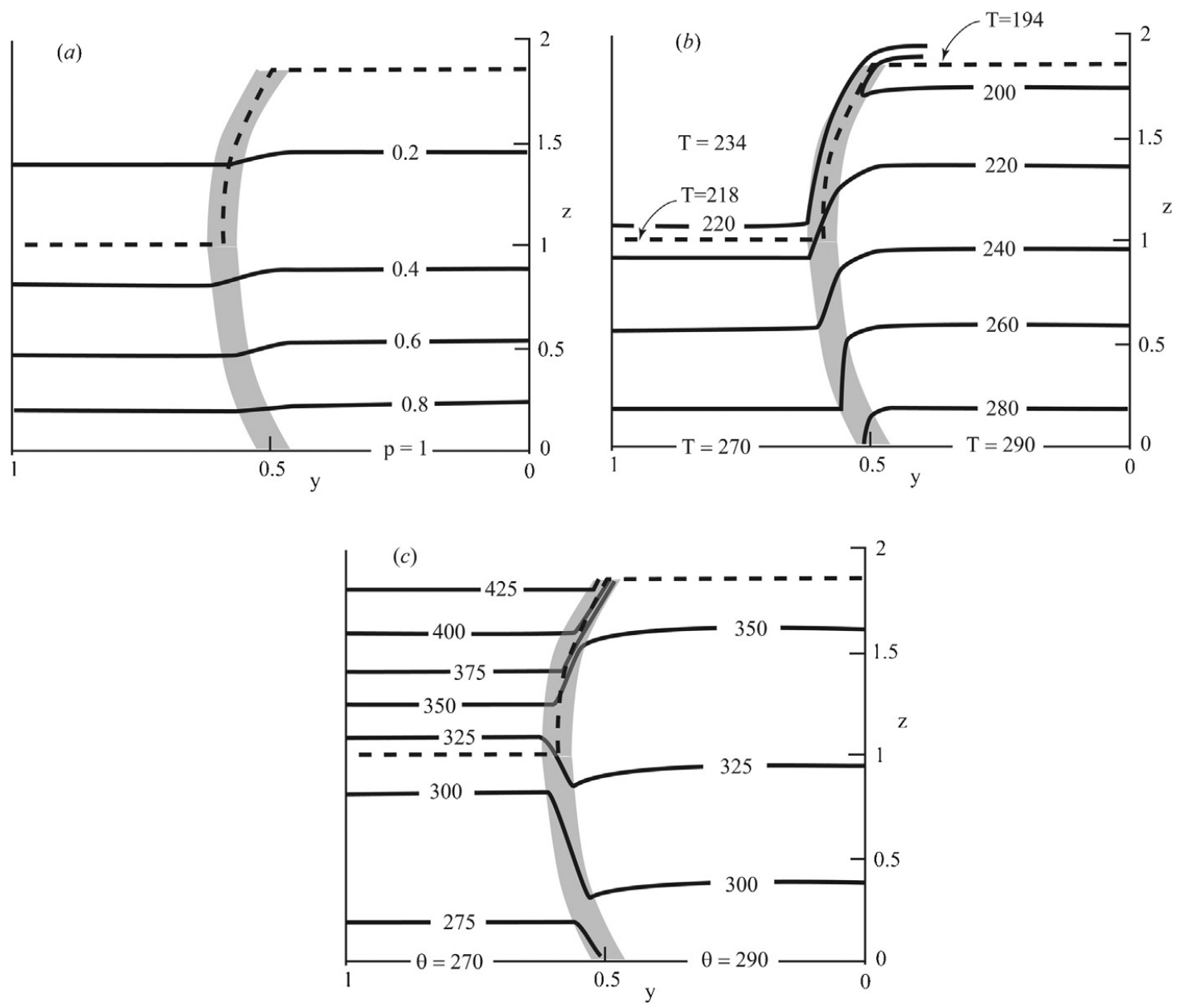

Fig. 4. (a) Isobaric surfaces calculated from the model (the surface pressure is scaled to unity); the dashed line marks the tropopause and the interface has been broadened (shaded line) to aid the visual display; (b) same as (a) but for the temperature; (c) same as (a) but for the potential temperature.

we assume a constant vertical diffusivity $v$ in the Ekman layer and a non-slip surface, so the Ekman flow is given by (Holton, 1979):

$$
u+i v=u_{\infty}\left(1-e^{-n z}\right)
$$

where the subscript $\infty$ denotes the free-tropospheric value and

$$
n \equiv(1+i)\left(\frac{f}{2 v}\right)^{1 / 2}
$$

More pertinent for capturing the surface branch of the meridional cell, we define the Ekman height by the first zero crossing in the vertical profile of the meridional wind, which is then given by

$$
h_{E}=\pi\left(\frac{2 v}{f}\right)^{1 / 2} \text {. }
$$

With the observed vertical diffusivity decreasing from $\mathrm{O}\left(10 \mathrm{~m}^{2} \mathrm{~s}^{-1}\right)$ in the free troposphere (Plumb and Mahlman, 1987) to zero at the surface, we take $v \approx 2 \mathrm{~m}^{2} \mathrm{~s}^{-1}$ as its representative value in the Ekman layer and plot the Ekman height in Fig. 5 (the dash-dot line). It grows from $0.5 \mathrm{~km}$ at the pole to $2 \mathrm{~km}$ where it merges with the ITCZ, the latter assumed to extent to about 5 latitudinal degrees (see later discussion in this section).

Integrating (6.1) through the Ekman layer, the mean meridional wind (overhatted) can be derived:

$$
\hat{v}=(2 \pi)^{-1} u_{\infty},
$$




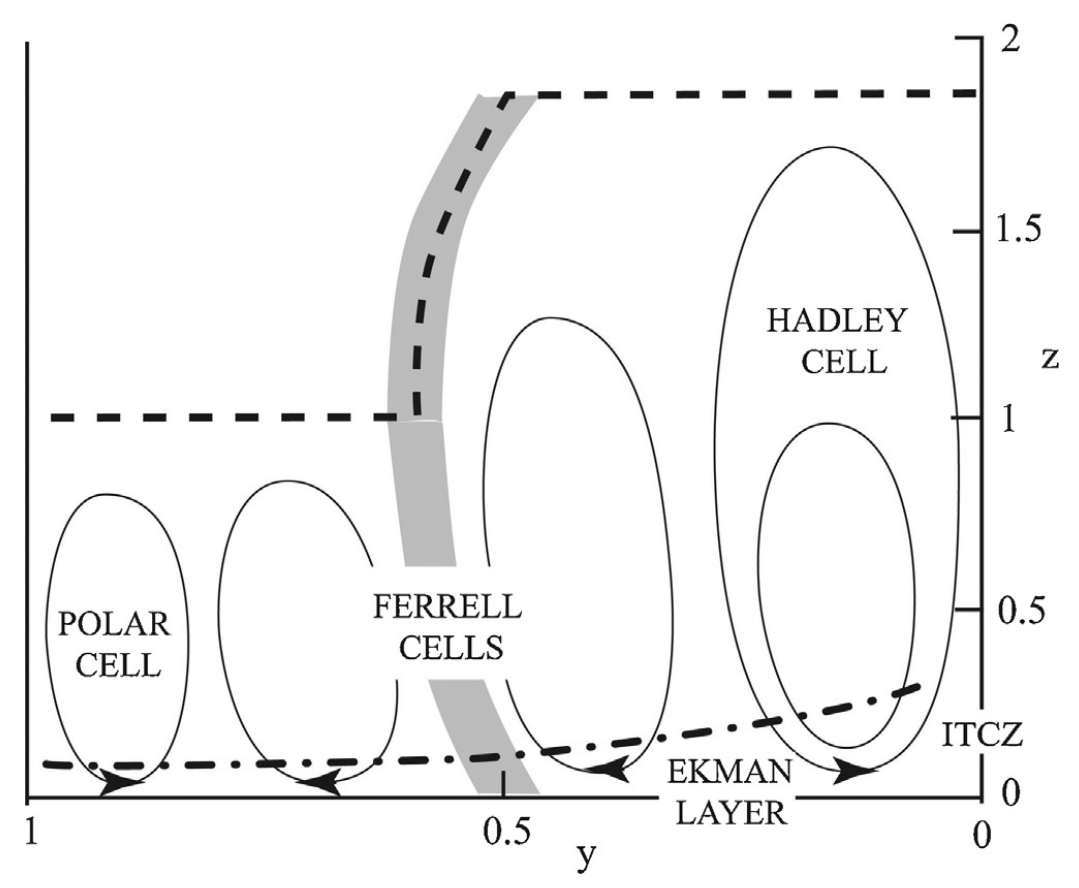

Fig. 5. A schematic of the meridional cells driven by the zonal wind via the Ekman dynamics. The dash-dotted line marks the top of the Ekman layer, which rises toward the equator and merges with the ITCZ. The cell boundaries are aligned with zero crossings of the zonal wind, resulting in the familiar cells as labeled except the Ferrel cell is bisected by the polar front into co-rotating sub-cells.

which simply tracks the overhead zonal wind - though weaker by an order-of-magnitude. Interestingly, this expression is independent of the uncertain vertical diffusivity hence a robust result. The Ekman transport, on the other hand, being a product of (6.3) and (6.4), does increase with the vertical diffusivity and with the rising Ekman height as one approaches the equator. With the above linkage, the latitudinal zero crossings of the zonal wind shown in Fig. 3 then divide the meridional cells, as sketched in Fig. 5, which are characterized by stronger surface branch and weak return flow distributed through the depth of the troposphere.

It is seen that the deduced meridional circulation contains familiar cells, but also new features. There is the Hadley cell driven by the easterly trades, whose surface convergence, being singular at the equator, may supply whatever the cumulus mass flux in the ITCZ. As the latter is the outcome of the global energy lance (Emanuel et al., 1994; Ou01, Ou07 and its Footnote 2), the above matching of the transport then specifies the mean latitudinal extent of the ITCZ. For a crude check, if one uses a cumulus mass flux of $2 \times 10^{11} \mathrm{~kg} \mathrm{~s}^{-1}$ (PO92, their Fig. 7.19) and easterly trades of $10 \mathrm{~m} / \mathrm{s}$, the ITCZ would extend to about 5 latitudinal degrees, not unlike that observed.

Adjacent to the Hadley cell is the Ferrel cell except it is now bisected by the polar front into two clockwise sub-cells (looking east), which will be referred as warm and cold Ferrel sub-cells. The warm sub-cell corresponds to the single Ferrel cell depicted in Rossby (1941), but in the more refined twocell scheme of Palmen (1951) (both are reproduced in PN69, their Figs. 4.1 and 4.2), the cold sub-cell rotates in the opposite sense from ours. In support of our scheme however, there is no observational evidence of an equatorward surface wind in mid-latitudes (PO92, Fig. 7.17), which moreover would require a non-existent mid-latitude easterly on account of the Ekman dynamics. Farther north, there is the polar cell driven by the polar easterly, but since the latter is a flitting feature (Section 4), so is the polar cell.

\section{Summary and discussion}

Completing a deductive theory of the earth's climate forced by the solar insolation, we consider here the general circulation of the atmosphere (part 1 ) to be followed by that of the ocean (part 2 ). With the MEP being a key closure assumption of the theory, we first reiterate our perspective of the MEP as 
rooted in the turbulence of the climatic fluids, which renders moot some recent questions raised on its application. We then recount the essential steps leading to the reduction of both planetary fluids to two thermal masses and the determination of their temperature and their dividing latitude at the ocean surface. Since no explicit dynamics was invoked, these properties are justifiably posed as the prior thermal constraint for the present dynamical derivation. In the following, we shall summarize the main findings and discuss their implications, particularly in their contrast to the existing views of the GAC.

\subsection{Zonal wind}

With the troposphere reduced to two thermal masses, we draw upon material conservation of the columnar PV to argue for its homogenization in thermal masses, which moreover is of zero value in the tropical air mass because of the hemispheric symmetry - a configuration that is supported by observational analyses. Inverting this PV, we reproduce the banded pattern of the prevailing wind: the easterly trades, the mid-latitude surface westerly, a weak polar easterly and a strong polar jet stream above the polar front. Although the model wind represents an upper-bound, it is nonetheless commensurate with the observed strength, suggesting that the GAC may be simply explained by the model physics. More broadly, with the central role played by eddy mixing of the PV, we interpret the GAC as the maximum macroscopic motion extractable by random eddies - within the confine of the thermal differentiation.

Since the polar jet stream and easterly trades are manifestations of the same zero PV of the tropical air mass, they are both eddy-driven and there is no need to invoke different generation mechanisms. The observed uniformity of the tropical angular momentum is not due to its conservation by the upper branch of the Hadley cell, which is too weak for that purpose (Jeffreys, 1933; Schneider, 2006); rather, it is simply the first integral of the zero PV, and the latter is the outcome of maximal eddy mixing a diametrically opposite regime from the axisymmetry. With a tropical air mass contiguous to midlatitudes, there is little justification for an energetically closed Hadley cell (Held and Hou, 1980; Satoh, 1994). Although PV mixing has been widely proposed in the generation of zonal jets (Baldwin et al., 2007), the difference here is that it is constrained by the thermal boundaries to yield the observed prevailing wind; the latter thus is unrelated to Rossby elasticity or Rhines scale (Baldwin et al., 2007; Dritschel and McIntyre, 2008).

Central to our GAC is the presence of an internal thermal - hence dynamical - boundary where the eddy diffusivity is strongly curtailed; one thus may not assume globally uniform eddy diffusivity - even as a first approximation - in calculating the zonal wind (Green, 1970). Moreover, since the emergence of this internal boundary is the outcome of the MEP - a global property, it may not be feasible to formulate a local closure theory of the turbulence, an often-stated goal in dynamical discussion (Held, 1999). Through the dynamical closure, we see that the prior thermal constraint has retained sufficient degrees of freedom to accommodate all dynamical requirements, and with the frictional dissipation already accounted for by the MEP, the GAC thus has little influence on the prior thermal constraint, as alluded to in Section 2.

Through the dynamical derivation, we see that the PV mixing provides in effect the only dynamical principle needed to explain the GAC and, as we shall see in part 2, it may explain the general ocean circulation as well. This dynamical unification of seemingly unrelated flows in dissimilar planetary fluids constitutes perhaps the strongest support of the PV mixing.

\subsection{Tropopause height}

Given the atmospheric weight, even the upper-bound GAC only slightly perturbs the surface pressure; together with the pressure equalization high aloft because of the rarefaction of the air, we deduce a tropical tropopause that is higher and colder than its polar counterpart. Having determined the polar front $(\mathrm{PF})$ in conjunction with the zonal wind, the above pressure equalization then fully specifies the tropospheric thermal fields, including the frontal zone and the extra-tropical tropopause (ET). The calculated fields bear close resemblance to the observed fields, which in fact downplays the GAC effect on the thermal fields except in the frontal zone. 
In our interpretation, the ET, just like the PF, is a quasi-material boundary to the eddy mixing hence should be marked by breaks in both the lapse rate and the IPV (Ambaum, 1997; Hoerling et al., 1991) and there is no need for additional generation processes (Ambaum, 1997; Haynes et al., 2001). On the other hand, although the mixing across the ET is curtailed, it would nonetheless manifest prominently in the cut-off eddies or isentropic filaments because of the strong thermal and PV contrasts (Shapiro et al., 1987; Pierrehumbert, 1991; Holton et al., 1995; Ambaum, 1997). It is recognized that the verticalaveraged lapse rate is quite smaller than the moist-adiabat in the extratropics, but this merely reflects the inversion at the polar front whereas the lapse rate in the embedding air masses remains moistadiabatic - the only rate that can be justified by moist convection; there is thus no need to invoke baroclinic neutrality (Stone, 1978; Held, 1982; Lindzen, 1993), which in any event is unsupported by GCM studies (Thuburn and Craig, 1997).

\subsection{Meridional circulation}

With the zonal wind independently constrained by the PV mixing, it then drives the meridional cells via the Ekman dynamics with its zero crossings marking the cell boundaries. There are the customary Hadley, Ferrel and polar cells except the Ferrel cell is bisected by the polar front into two co-rotating sub-cells. The preeminence of the Hadley cell stems from the singular Ekman convergence at the equator, which may supply the cumulus mass flux in the ITCZ as required by the global energy balance; the matching of the two transports in fact specifies the latitudinal extent of the ITCZ, which is comparable with that observed.

It is well recognized that radiative heating is too weak to account for the observed Hadley circulation (Schneider, 2006; Kim and Lee, 2001), a shortfall that was remedied by imposing the observed condensation heating (Schneider and Lindzen, 1977); but the latter amounts to specifying the cumulus mass flux - precisely the property that defines the strength of the Hadley circulation. In our formulation, this flux is the outcome of the global energy balance (Emanuel et al., 1994; Ou01; Ou07) and its concentration in the ITCZ simply reflects the singular Ekman convergence there, which can supply the required flux (only a small portion may be absorbed by other surface convergence zones). This singular Ekman transport may also explain the acute seasonal asymmetry of the Hadley circulation manifested only in its downward branch (Dima and Wallace, 2003). With the Hadley cell determined, the upper-level convergence at its poleward boundary could induce - in a more refined model - a subtropical front to drive a strong subtropical jet because of the rarefied air (above $200 \mathrm{mb}$ ). While this is a significant feature of the GAC, it cannot be addressed by our crude model.

There were many studies of the meridional circulation driven by the Eliassen-Palm flux (Eliassen, 1951; Chang, 1996) or the zonal wind (Kuo, 1956; Lorenz, 1967; Green, 1970; Kim and Lee, 2001), but unless these "forcings" can be independently constrained, they offer no causal explanation. The difference here is the physical closure of the global system, so causality can be established, which proceeds as follows: the thermodynamics principle MEP stemming from the microscopic turbulence produces the thermal boundaries, the implied PV mixing in air masses then induces the prevailing wind, which in turn drives the meridional circulation via the Ekman dynamics.

\subsection{GCM perspective}

Since the MEP stems from microscopic turbulence, it is operative in GCMs only if the latter have adequately resolved eddies, and then since the MEP describes the expectant macroscopic state, it represents an ensemble mean of the modeled states; both thus pose significant computational challenges. As the atmosphere is differentially heated mainly by the ocean, the PF is aligned with the oceanic front, a linkage that is indeed demonstrated by GCM calculations (Nakamura et al., 2008), and given the central role of the PF in constraining the GAC, the simulation of the latter by atmosphere-only GCM (AGCM) thus requires a proper specification of the observed sea-surface temperature.

For coupled GCMs, on the other hand, these thermal boundaries should emerge if the MEP is operative; this suggests that the often-used swamp ocean is inadequate in capturing the thermal state and hence the GAC. Questions have been raised about the rotation effect on the GAC, but with the midlatitude positioning of fronts the consequence only of the thermodynamics, the changing rotation 
may affect only the width of the frontal zone (via the deformation radius), which would nonetheless displace the core of the polar jet stream. On the other hand, because of the zero PV, the maximum jet speed is scaled by the product of this deformation radius and the mid-latitude Coriolis parameter hence may not be a strong function of the rotation rate. Considering the aforementioned MEP requirements, one may not use the AGCM output (for example, Del Genio and Suozzo, 1987) as an arbiter of the above deduction.

\section{Acknowledgments}

I want to thank the Lamont directorate for the salary support that has made this research possible.

\section{Appendix A. Method for calculating the zonal wind}

We describe here the method for solving the zonal wind governed by (4.1), (4.2) and (4.7) subjected to constraints (4.9)-(4.13). The first integral of (4.1) yields

$$
m_{1}=\frac{1}{2} y^{2}+m_{1,0} \text { for } 0<y<y_{2}
$$

with the zonal wind at the equator $m_{1,0}$ an unknown integration constant. Similarly, integrating (4.2) outside the frontal zone where $h_{2}=1$ (4.10) and applying (4.11) yields

$$
m_{2}=\frac{1}{2}\left(y^{2}-1\right)-P_{2}(y-1) \text { for } y>y_{2},
$$

which contains the unknown PV $\left(P_{2}\right)$ of the polar air mass. In the frontal zone, we define

$$
\hat{m}=m_{1}-m_{2}
$$

so (4.1) and (4.2) can be combined to yield

$$
\frac{d \hat{m}}{d y}=P_{2} h_{2},
$$

and (4.7) becomes

$$
\frac{d h_{2}}{d y}=\frac{1}{\varepsilon} \cdot \frac{y}{1-y^{2}} \hat{m}
$$

To obtain the solution, we guess the values of $m_{1,0}$ and $P_{2}$, then use the conditions (4.9) and (4.13) to integrate (A.4) and (A.5) from $y_{1}$ to $y_{2}$ defined by (4.10), at which point we check the condition (4.12) and the matching of $m_{2}$ to that of (A.2). The initial guess can be systematically adjusted until these conditions are satisfied to the desired accuracy. For the solution shown in Fig. 3, the integration is based on a fourth-order Runge-Kutta scheme, the grid spacing in $y$ is $10^{-2}$, and the required conditions are satisfied to within $10^{-3}$.

\section{Appendix B. Method for calculating the thermal field}

For the double-subscripted variables used here, the first subscript denotes the air masses ( 1 for tropical and 2 for polar, indicated by the open circles in Fig. 1) and the second subscript denotes the levels ( 0 for the sea surface, 1 for the polar front, 2 for the tropopause and 3 for the highest level reached by the tropopause, marked by open squares in Fig. 1). Within air masses, we have, see (4.5),

$$
d \ln p=g\left(\gamma R_{d}\right)^{-1} d \ln T ;
$$

and for the isothermal stratosphere of temperature $T$, we have

$$
d \ln p=-\frac{g d z}{R T} .
$$

As an approximation, we assume the pressure to be equalized at the surface and level 3 , the latter can be checked a posteriori, and to determine the tropopause properties outside the frontal zone, we 
integrate (B.1) and (B.2) between these two levels over the tropical (lhs) and polar (rhs) regions to yield

$$
\ln \frac{T_{12}}{T_{10}}=\ln \frac{T_{22}}{T_{20}}-\frac{\left[\left(T_{10}-T_{12}\right)-\left(T_{20}-T_{22}\right)\right]}{T_{23}}
$$

where $T_{23}$ denotes the temperature of the isothermal stratosphere (see Fig. 1). Assuming the scaled width of the frontal zone to be small compared with unity, the known planetary skin temperature $T_{\text {skin }}$ imposes a constraint that

$$
T_{\text {skin }}=y_{1} T_{12}+\left(1-y_{1}\right) T_{22} \text {. }
$$

Since the ET and PF slopes in the opposite sense (see Fig. 2 and discussion in PN69, their Section 7.4), so is the temperature jump across them, which is thus set to zero at the wedge, or

$$
T_{23}=T_{1 w},
$$

and the subscript " $1 w$ " indicates the tropical air mass at the wedge (see Fig. 1). Combined with the matching of the pressure at this wedge that states, see (4.5)

$$
\ln \left(\frac{T_{22}}{T_{20}}\right)=\ln \left(\frac{T_{1 w}}{T_{10}}\right)
$$

we have then

$$
T_{23}=T_{22}\left(\frac{T_{10}}{T_{20}}\right) \text {. }
$$

With (B.7) and given the known values of $y_{1}, T_{\text {skin }}, T_{10}$ and $T_{20}$ (all determined previously), (B.3) and (B.4) then form a closed set governing the unknown tropopause temperature $T_{12}$ and $T_{22}$ outside the frontal zone.

To further simplify the mathematics, we denote the surface and tropopause temperature ranges by the symbols

$$
\begin{aligned}
& \Delta T_{0}=T_{10}-T_{20}, \\
& \Delta T_{2}=T_{12}-T_{22},
\end{aligned}
$$

then, from (B.4), the unknown tropopause temperature can be expressed as

$$
\begin{aligned}
& T_{12}=T_{\text {skin }}+\left(1-y_{1}\right) \Delta T_{2}, \\
& T_{22}=T_{\text {skin }}-y_{1} \Delta T_{2},
\end{aligned}
$$

and (B.3) is reduced to an equation containing a single unknown $\Delta T_{2}$, the tropopause temperature range. It thus can be solved by simply guessing an initial value and adjusting it systematically until the equation is satisfied. With $\Delta T_{2}$ known, one may then calculate the tropopause temperature as well as temperature and pressure profiles outside the frontal zone.

With the stratosphere temperature $T_{23}$ now known from (B.7), we may calculate the thermal profiles in the frontal zone by integrating (B.1) and (B.2) from the ground up, punctuated by the PF known from Fig. 3, and adjust the ET height to satisfy the pressure equalization condition at the upper level 3.

\section{References}

Ambaum, M., 1997. Isentropic formation of the tropopause. J. Atmos. Sci. 54, 555-568.

Baldwin, M.P., Rhines, P.B., Huang, H.-P., McIntyre, M.E., 2007. The jet-stream conundrum. Science 315, 467-468.

Bruers, S., 2006. Classification and discussion of macroscopic entropy production principles. arXiv:cond-mat/0604482.

Chang, E.K.M., 1996. Mean meridional circulation driven by eddy forcings of different timescales. J. Atmos. Sci. 53, $113-125$.

Cushman-Roisin, B., 1994. Introduction to Geophysical Fluid Dynamics. Prentice Hall, Upper Saddle River, New Jersey, USA, pp. 320.

Del Genio, A.D., Suozzo, R.J., 1987. A comparative study of rapidly and slowly rotating dynamical regimes in a terrestrial general circulation model. J. Atmos. Sci. 44 (6), 973-986. 
Dewar, R., 2003. Information theory explanation of the fluctuation theorem, maximum entropy production and self-organized criticality in non-equilibrium stationary states. J. Phys. A: Math. Gen. 36, 631-641.

Dewar, R.C., 2005. Maximum entropy production and nonequilibrium statistical mechanics. In: Kleidon, A., Lorenz, R.D. (Eds.), Non-equilibrium Thermodynamics and the Production of Entropy: Life, Earth, and Beyond. Springer, Heidelberg.

Dima, I.M., Wallace, J.M., 2003. On the seasonality of the Hadley cell. J. Atmos. Sci. 60, 1522-1527.

Dritschel, D.G., McIntyre, M.E., 2008. Multiple jets as PV staircases: the Phillips effect and the resilience of eddy transport barriers. J. Atmos. Sci. 65, 855-874.

Eliassen, A., 1951. Slow thermally or frictionally controlled meridional circulation in a circular vortex. Astrophys. Norv. 5, 19-60.

Emanuel, K.A., Neelin, J.D., Bretherton, C.S., 1994. On large-scale circulations in convecting atmospheres. Q. J. R. Meteorol. Soc. $120,1111-1143$.

Goody, R., 2007. Maximum entropy production in Climate Theory. J. Atmos. Sci. 64, 2735-2739.

Green, J.S.A., 1970. Transfer properties of the large-scale eddies and the general circulation of the atmosphere. Q. J. R. Meteorol. Soc. 96 (408), 157-185.

Grinstein, G., Linsker, R., 2007. Comments on a derivation and application of the 'maximum entropy production' principle. J. Phys. A: Math. Theor. 40, 9717-9720.

Hadley, G., 1735. Concerning the cause of the general trade-winds. Philos. Trans. 39, 58-62.

Hartmann, D.L., 1994. Global Physical Climatology. Academic Press, San Diego, California, USA, pp. 411.

Haynes, P., Shuckburgh, E., 2000. Effective diffusivity as a diagnostic of atmospheric transport: 2. Troposphere and lower stratosphere. J. Geophys. Res. 105 (D18), 22795-22810.

Haynes, P., Scinocca, J., Greenslade, M., 2001. Formation and maintenance of the extratropical tropopause by baroclinic eddies. J. Geophys. Res. 28 (22), 4179-4182.

Held, I.M., 1982. On the height of the tropopause and the static stability of the troposphere. J. Atmos. Sci. 39, $412-417$.

Held, I.M., 1999. The macroturbulence of the troposphere. Tellus 51A-B, 59-70.

Held, I.M., Hoskins, B.J., 1985. Large-scale eddies and the general circulation of the troposphere. Adv. Geophys. 28A, 3-31.

Held, I.M., Hou, A.Y., 1980. Nonlinear axially symmetric circulations in a nearly inviscid atmosphere. J. Atmos. Sci. 37, 515-533.

Hoerling, M.P., Shaack, T.K., Lenzen, A.J., 1991. Global objective tropopause analysis. Mon. Weather Rev. 119, $1816-1831$.

Holton, J.R., 1979. An Introduction to Dynamic Meteorology, 2nd ed. Academic Press, pp. 391.

Holton, J.R., Haynes, P.H., Mcintyre, M.E., Douglass, A.R., Rood, R.B., Pfister, L., 1995. Stratosphere-troposphere exchange. Rev. Geophys. 33, 403-439.

Hoskins, B.J., 1991. Towards a PV-theta view of the general circulation. Tellus 43AB, 27-35.

Hoskins, B.J., McIntyre, M.E., Robertson, A.W., 1985. On the use and significance of isentropic potential vorticity maps. Q. J. R. Meteorol. Soc. 111, 877-946.

Jeffreys, H., 1926. On the dynamics of geostrophic winds. Q. J. R. Meteorol. Soc. 52, 85-104.

Jeffries, M., 1933. The function of cyclones in the general circulation (reprinted in Theory of Thermal Convection, Dover, 1962).

Kim, H.-K., Lee, S., 2001. Hadley cell dynamics in a primitive equation model. Part II: Nonaxisymmetric flow. J. Atmos. Sci. 58, $2859-2871$.

Kleidon, A., 2009. Non-equilibrium thermodynamics and maximum entropy production in the Earth system: applications and implications. Naturwissenschaften 96, 653-677.

Kuo, H.-L., 1956. Forced and free meridional circulations in the atmosphere. J. Meteorol. 13, 561-568.

Li, J., 2009. On the extreme of internal entropy production. J. Phys. A: Math. Theor. 42 (035002), http://dx.doi.org/10.1088/1751-8113/42/3/035002.

Lindzen, R.S., 1993. Baroclinic neutrality and the tropopause. J. Atmos. Sci. 50 (8), 1148-1151.

Lorenz, E.N., 1967. The nature and Theory of the General Circulation of the Atmosphere. World Meteorological Organization, Geneva, $161 \mathrm{pp}$

Lucarini, V., Fraedrich, K., Ragone, F., 2011. New results on the thermodynamic properties of the climate. J. Atmos. Sci. 68, $2438-2458$

Martyushev, L.M., Seleznev, V.D., 2006. Maximum entropy production principle in physics, chemistry and biology. Phys. Rep. 426, 1-45.

Nakamura, N., Sampe, T., Goto, A., Ohfuchi, W., Xie, S.-P., 2008. On the importance of midlatitude oceanic frontal zones for the mean state and dominant variability in the tropospheric circulation. Geophys. Res. Lett. 35 (L15709), http://dx.doi.org/10.1029/2008GL034010.

Noda, A., Tokioka, T., 1983. Climates at minima of the entropy exchange rate. J. Meteorol. Soc. Jpn. 61 (6), 894-908.

Obrien, D.M., Stephens, G.L., 1995. Entropy and climate. 2. Simple models. Q. J. R. Meteorol. Soc. 121 (527), 1773-1796.

Ou, H.W., 2001. Possible bounds on the earth's surface temperature: from the perspective of a conceptual global-mean model. J. Clim. 14, 2976-2988.

Ou, H.W., 2006. Meridional thermal field of a coupled ocean-atmosphere system: a conceptual model. Tellus 58A, 404-415.

Ou, H.W., 2007. Hydrological cycle and ocean stratification in a coupled climate system: a theoretical study. Tellus 59A, 683-694.

Ozawa, H., Ohmura, A., Lorenz, R.D., Pujol, T., 2003. The second law of thermodynamics and the global climate system: a review of the maximum entropy production principle. Rev. Geophys. 41 (4), 10182003.

Palmen, E., 1951. The role of atmospheric disturbances in the general circulation. Q. J. R. Meteorol. Soc. 77, 337-354.

Palmen, E., Newton, C.W., 1969. Atmospheric Circulation System. Academic Press, New York, USA, pp. 606.

Paltridge, G.W., 1975. Global dynamics and climate - a system of minimum entropy exchange. Q. J. R. Meteorol. Soc. 101, 475-484.

Pascale, S., Gregory, J.M., Ambaum, M.H.P., Tailleux, R., Lucarini, V., 2012. Vertical and horizontal processes in the global atmosphere and the maximum entropy production conjecture. Earth Syst. Dyn. 3, 19-32.

Pauluis, O., Held, I.M., 2002. Entropy budget of an atmosphere in radiative-convective equilibrium. Part I: Maximum work and frictional dissipation. J. Atmos. Sci. 59 (2), 125-139.

Peixoto, J.P., Oort, A.H., 1992. Physics of Climate. American Institute of Physics, College Park, Maryland, USA, pp. 520.

Peixoto, J.P., Oort, A.H., de Almeida, M., Tome, A., 1991. Entropy budget of the atmosphere. J. Geophys. Res. 96, 10981-10988.

Pierrehumbert, R.T., 1991. Large-scale horizontal mixing in planetary atmospheres. Phys. Fluids A 3 (5), 1250-1260. 
Platzman, G.W., 1949. The motion of barotropic disturbances in the upper troposphere. Tellus 1, 53-64.

Plumb, R.A., Mahlman, J.D., 1987. The zonally averaged transport characteristics of the GFDL General Circulation/Transport Model. J. Atmos. Sci. 44 (2), 298-327.

Prigogine, I., 1955. Introduction to Thermodynamics of Irreversible Processes. Thournes, Chicago.

Rossby, C.-G., 1941. The scientific basis of modern meteorology. In: Hambidge, G. (Ed.), Yearbook of Agriculture, Climate and Man. U.S. Government Printing Office, Washington, DC, pp. 599-655.

Rossby, C., 1947. On the distribution of angular velocity in gaseous envelopes under the influence of large-scale horizontal mixing process. Bull. Am. Meteorol. Soc. 28, 53-68.

Satoh, M., 1994. Hadley circulations in radiative-convective equilibrium in an axially symmetric atmosphere. J. Atmos. Sci. 51, 1947-1968.

Schneider, T., 2006. The general circulation of the atmosphere. Annu. Rev. Earth Planet. Sci. 34, 655-688.

Schneider, E.K., Lindzen, R.S., 1977. Axially symmetric steady-state models of the basic state for instability and climate studies. Part I. Linearized calculations. J. Atmos. Sci. 34, 263-279.

Shapiro, M.A., Hampel, T., Krueger, A.J., 1987. The arctic tropopause fold. Mon. Weather Rev. 115, 444-454.

Starr, V.P., 1968. Physics of Negative Viscosity Phenomena. McGraw-Hill, New York, USA, pp. 256.

Stone, P.H., 1978. Baroclinic adjustment. J. Atmos. Sci. 35, 561-571.

Swanson, K.L., 2001. Upper-tropospheric potential vorticity fluctuations and the dynamical relevance of the time mean. J. Atmos. Sci. 58, 1815-1826.

Taylor, G.I., 1915. Eddy motion in the atmosphere. Philos. Trans. R. Soc. Lond. A 140, 1-26.

Thuburn, J., Craig, G., 1997. GCM tests of theories for the height of the tropopause. J. Atmos. Sci. 54, 869-882.

Virgo, N., 2010. From maximum entropy to maximum entropy production: a new approach. Entropy 12, 107.

Webster, P.J., 1994. The role of hydrological processes in ocean-atmosphere interactions. Rev. Geophys. 32, 427-476. 